Deivisson Freitas da Silva ${ }^{a}$

José Tavares-Neto ${ }^{a}$

Rita Franco Rêgo ${ }^{a}$

\section{Lesões oculares em trabalhadores da pesca comercial: uma revisão de literatura}

\author{
Ocular injuries in commercial fishing workers: \\ a literature review
}

a Universidade Federal da Bahia, Faculdade de Medicina da Bahia. Salvador, Bahia, Brasil.

Contato:

Deivisson Freitas da Silva

E-mail:

deivissonfs@gmail.com

Os autores declaram que este trabalho não foi subvencionado e que não apresenta conflitos de interesses.

Trabalho baseado na monografia de curso de Deivisson Freitas da Silva intitulada "Lesões oculares em trabalhadores da pesca: mais uma história de pescador?", defendida em 2013 na Faculdade de Medicina da Bahia, da Universidade Federal da Bahia.

O trabalho não foi apresentado em evento científico.

Recebido: 15/10/2015

Revisado: 25/05/2016

Aprovado: 30/05/2016

\section{Resumo}

Introdução: a pesca é uma atividade perigosa, associada à elevada morbimortalidade. Entretanto, são escassos os estudos sobre lesões oculares em trabalhadores desta atividade. Objetivo: identificar lesões oculares relacionadas à exposição ocupacional na pesca comercial e possíveis fatores de risco e de proteção relacionados. Métodos: revisão de literatura nas bases bibliográficas MedLine, LILACS-BIREME, SCOPUS, Web of Science e Cochrane, utilizando os termos de busca "pesca" e "lesão ocular". Foram incluídos: artigos completos disponíveis escritos em inglês, português ou espanhol, publicados de abril de 1993 a abril de 2013; e estudos em seres humanos. Resultados: foram incluídos 18 artigos, provenientes de 12 países. O desenho de estudo mais prevalente foi o de corte transversal. Discussão: as lesões mais frequentes se relacionavam com a exposição solar excessiva, como a catarata e a degeneração macular senil, e com traumas oculares, principalmente as lesões perfurantes pelo gancho de pesca ou por fragmentos de animais marinhos. Esses agravos foram relacionados com fatores de risco como o manuseio de peixes e frutos do mar e as jornadas prolongadas de trabalho. De acordo com os estudos, a maioria das doenças oftalmológicas relacionadas ao trabalho da pesca é prevenível, o que indica a necessidade de políticas de promoção de saúde direcionadas para a população envolvida com esse ramo.

Palavras-chave: saúde do trabalhador; indústria pesqueira; oftalmopatias; pesca; ferimentos e lesões.

\begin{abstract}
Introduction: commercial fishing is a dangerous activity which is associated with high morbimortality. However, there are few studies on fishing workers' eye injuries. Objective: to identify occupational ocular injuries in commercial fishing and possible related risk and protection factors. Methods: literature review on the databases MedLine, LILACS-BIREME, SCOPUS, Web of Science, and Cochrane through the searching terms "fishing" and "ocular/eye injury". Both were taken into account: full articles available in English, Portuguese or Spanish, published from April 1993 to April 2013, and research involving human beings. Results: 18 articles from 12 countries were included. Most studies were cross-sectional. Discussion: the most frequent injuries were due to excessive solar exposure, such as cataract and senile macular degeneration, and to ocular trauma, mainly perforations by fishhook or by fragments of marine animals. These injuries were associated with risk factors like fish and other seafood handling and long working time. According to the studies, most of the eye injuries related to fishing can be prevented, indicating the need for health promoting policies aimed at the population involved in this activity.
\end{abstract}

Keywords: occupational health; fishing industry; ophthalmopathy; commercial fishing; wounds and injuries. 


\section{Introdução}

Existem diversas formas de conceituar o termo "pesca”. De acordo com o glossário da Food and Agriculture Organization das Nações Unidas (FAO), a pesca é atividade que leva à colheita de peixes, nas fontes hídricas naturais ou por meio da aquicultura $^{1}$; a legislação brasileira amplia essa definição, caracterizando-a por meio de vários procedimentos (e.g., retirada, extração, coleta, apanhamento, apreensão ou captura) de espécies de algumas ordens taxonômicas (e.g., peixes, crustáceos, moluscos e vegetais hidróbios, com ou sem aproveitamento econômico $)^{2}$. Descrita entre as atividades primitivas da espécie humana, passou por transformações radicais ao longo dos séculos, expandindo-se desde a pesca de subsistência com instrumentos rudimentares até a pesca em grande escala com amplo suporte tecnológico ${ }^{3}$.

Essa atividade é frequentemente definida em relação a alguns ou todos os seguintes critérios: pessoas envolvidas, espécie ou tipo de peixe, área de água ou fundo do mar, método de pesca, classe de barcos e finalidade da atividade. O Ministério da Agricultura, Pecuária e Abastecimento no Brasil classifica a atividade segundo a finalidade econômica e social em artesanal, amadora e industrial ${ }^{4}$. Nesse sentido, a pesca comercial é predominantemente uma atividade industrial, embora o pescador artesanal também costume realizar atividade de comércio do pescado, enquanto a pesca amadora é predominantemente para fins recreativos/esportivos.

Segundo o ministério, o Brasil conta com aproximadamente 1 milhão de pescadores artesanais e 9 mil trabalhadores da pesca industrial, responsáveis pela captura de 803 toneladas de pescado no ano de 2011, sendo aproximadamente 70\% em águas marinhas ${ }^{5}$. Também denominada de pesca em pequena escala, a pesca artesanal responde por aproximadamente $45 \%$ dessa produção. A região com maior produção é o Nordeste, apesar do estado com maior produção ser Santa Catarina, na região $\mathrm{Sul}^{5}$.

Em contraste com seu caráter milenar, só em 2009 a legislação brasileira regulamentou os diversos aspectos relacionados à pesca e a seu exercício profissional, por meio da Lei $\mathrm{n}^{\mathrm{o}} 11.959$, de 29 de junho de $2009^{6}$. A atividade pesqueira é associada a elevadas taxas de morbidade, de letalidade e de graves lesões decorrentes da atividade ${ }^{7,8}$, em grande parte advindas de fatores intervenientes, de ampla heterogeneidade e naturezas, desde o ambiente de trabalho hostil, tempo de confinamento prolongado, desempenho de funções fisicamente estafantes, exposição a longas jornadas de trabalho e climas extremos, até fatores sociais, econômicos e culturais ${ }^{9}$. Não obstante, houve pouco progresso na incorporação desses conhecimentos às práticas governamentais ao redor do mundo; e, seguindo essa tendência mundial, também no Brasil ainda não houve precisa identificação e resolução dos problemas de saúde relativos à atividade pesqueira, bem como na operacionalização dos serviços e pesquisas em saúde nessa área.

Há três décadas, um estudo ${ }^{10}$ descreveu em pescadores do Reino Unido quatro vezes mais chances de morrer do que os trabalhadores das minas de carvão em razão de acidentes no trabalho. De modo complementar, outro estudo sobre os riscos da pesca comercial $^{11}$ constatou grande proporção de mortes preveníveis. Na Noruega, pesquisadores relataram em pescadores a terceira maior taxa de custo anual por lesões ocupacionais ${ }^{12}$. Outro trabalho ${ }^{13}$ descreveu os motivos de busca por atendimento médico pelos pescadores, sendo 35\% traumas e $65 \%$ outras doenças. Dentre os traumas, em razão do manuseio de peixes ou instrumentos, os mais frequentes foram: contusões, cortes, perfurações, fraturas e entorses, principalmente em mãos, dedos, membros superiores e olhos ${ }^{9,11-13}$, enquanto doenças prevaleceram as digestivas, respiratórias, dermatológicas e oftalmológicas $^{9-13}$. Contudo, há poucos estudos sobre as lesões relacionadas ao trabalho da pesca, em especial as oculares.

Diante da relevância social e econômica do pescador comercial e da evidente necessidade da elaboração de um panorama da sua saúde ocupacional, notabiliza-se a demanda por estudos de revisão que possibilitem esse diagnóstico. Nesse ínterim, no que concerne especificamente à saúde ocular dessa categoria ocupacional, existem apenas estudos voltados à análise de patologias/associações específicas. Assim, este estudo teve como objetivo revisar a literatura, identificando as lesões oculares relacionadas à exposição ocupacional no exercício da atividade da pesca comercial, buscando-se identificar possíveis fatores de risco e de proteção relacionados a essas lesões.

\section{Métodos}

O estudo foi realizado com dados secundários por meio de uma revisão da literatura nos seguintes bancos de dados on-line: MedLine, Cochrane, Web of Science, LILACS-BIREME e SCOPUS.

As estratégias de busca adotadas para cada uma das bases foram fundamentadas nos termos listados no Quadro 1, organizados da seguinte maneira:

1. MedLine: (\#1) AND (\#2) NOT (\#3[Author])

2. Cochrane: (\#1) and (\#2) 
3. Web of Science: Topic $=(\# 1)$ AND Topic $=(\# 2)$ NOT Author $=(\# 3)$

4. LILACS-BIREME: (\#1) AND (\#2) [Words] AND NOT (\#3) [Author]

5. SCOPUS: TITLE-ABS-KEY((\#1) AND (\#2))

Quadro 1 Termos utilizados nas estratégias de buscas nas bases de dados

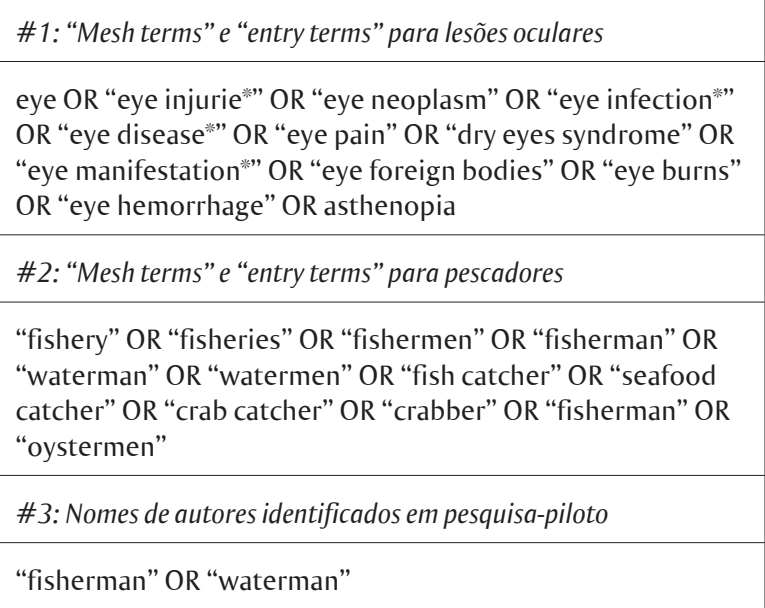

A busca pelos termos foi restringida aos títulos, resumos e palavras-chave. Em seguida, foram aplicados os seguintes critérios de inclusão (limites):

1. Data de publicação: entre 14 de abril de 1993 e 14 de abril de 2013;

2. Espécie estudada: somente estudos em seres humanos;

3. Idioma em que o artigo foi escrito: português, inglês ou espanhol;

4. Disponibilidade do resumo para leitura: resumo disponível.

Com a leitura dos títulos e resumos, foram selecionados apenas os estudos contendo a discussão da associação entre o exercício da pesca comercial e o desenvolvimento/risco de lesões oftalmológicas. Todos os estudos incluídos tiveram suas referências verificadas em busca de estudos elegíveis adicionais. Sites com vinculação institucional e especializada também foram utilizados como fontes de busca.

As referências e os dados relevantes (autoria, ano, país, tipo de estudo, população estudada e objetivos) de cada artigo foram obtidos e inseridos em uma tabela do software Microsoft Excel para serem resumidos e analisados descritivamente.

Para esse tipo de estudo, não há necessidade de análise por Comitê de Ética em Pesquisa (CEP), segundo o regramento estabelecido na resolução $\mathrm{n}^{\circ}$ 466, de dezembro de 2012, do Conselho Nacional de Saúde.

\section{Resultados}

Foram aplicadas as estratégias e limites de busca nas bases citadas, tendo sido encontrados 152 artigos: 51 na MedLine; 37 na Cochrane; 18 na SCOPUS; 17 na Web of Science; e 29 na LILACS-BIREME. Após a leitura dos resumos, levando-se em consideração a população-alvo (pescadores) e as variáveis a serem pesquisadas (lesões oculares, etiologia das lesões oculares e medidas de prevenção), foram selecionados 40 artigos: 18 na MedLine, 10 na SCOPUS, 10 na Web of Science e dois na LILACS-BIREME; nenhum artigo foi selecionado na Cochrane. Excluindo-se os 15 artigos repetidos nos resultados das diferentes bases, foram selecionados 25 artigos; no entanto, apenas 20 foram obtidos por download na rede da Universidade Federal da Bahia. Os cinco artigos não obtidos não foram encontrados mesmo por meio de buscas pelos respectivos códigos de identificação (PMID, DOI); eram exclusivamente pagos e/ou o e-mail para contato com os autores não estava disponível. Assim, foram obtidos 20 artigos para a leitura completa a partir do contato com o autor principal. Após sua leitura, dois estudos foram excluídos da revisão ${ }^{14,15}$, pois a população estudada (pescadores esportivos e pescadores não comerciais) diferia da população-alvo pesquisada (pescadores comerciais), o que reduziu para 18 o número de artigos que efetivamente compuseram a revisão de acordo com o fluxograma de seleção dos artigos apresentado na Figura 1.

A sistematização das informações colhidas nos artigos se processou por meio do preenchimento de uma tabela-padrão, que se encontra em sua forma resumida no Quadro 2, e apresenta as características gerais dos estudos (autoria, ano, país, tipo de estudo, população estudada e objetivos).

Dos dezoito artigos obtidos, onze (61\%) foram escritos na última década - após 2005. Encontrou-se uma ampla distribuição mundial: quatro foram desenvolvidos nos Estados Unidos da América; três na Croácia; dois na Austrália; e os demais países possuíam um artigo cada (Brasil, China, Cuba, Espanha, Grécia, Índia, Itália, Noruega e Turquia). Quanto ao tipo de estudo, foram obtidos sete cortes transversais, cinco relatos de caso (sendo que dois destes incluíam revisões de literatura), quatro revisões de literatura e duas coortes (uma prospectiva e uma retrospectiva). Os detalhes desses artigos são apresentados em seguida, obedecendo à ordem cronológica de publicação (Quadro 2). 


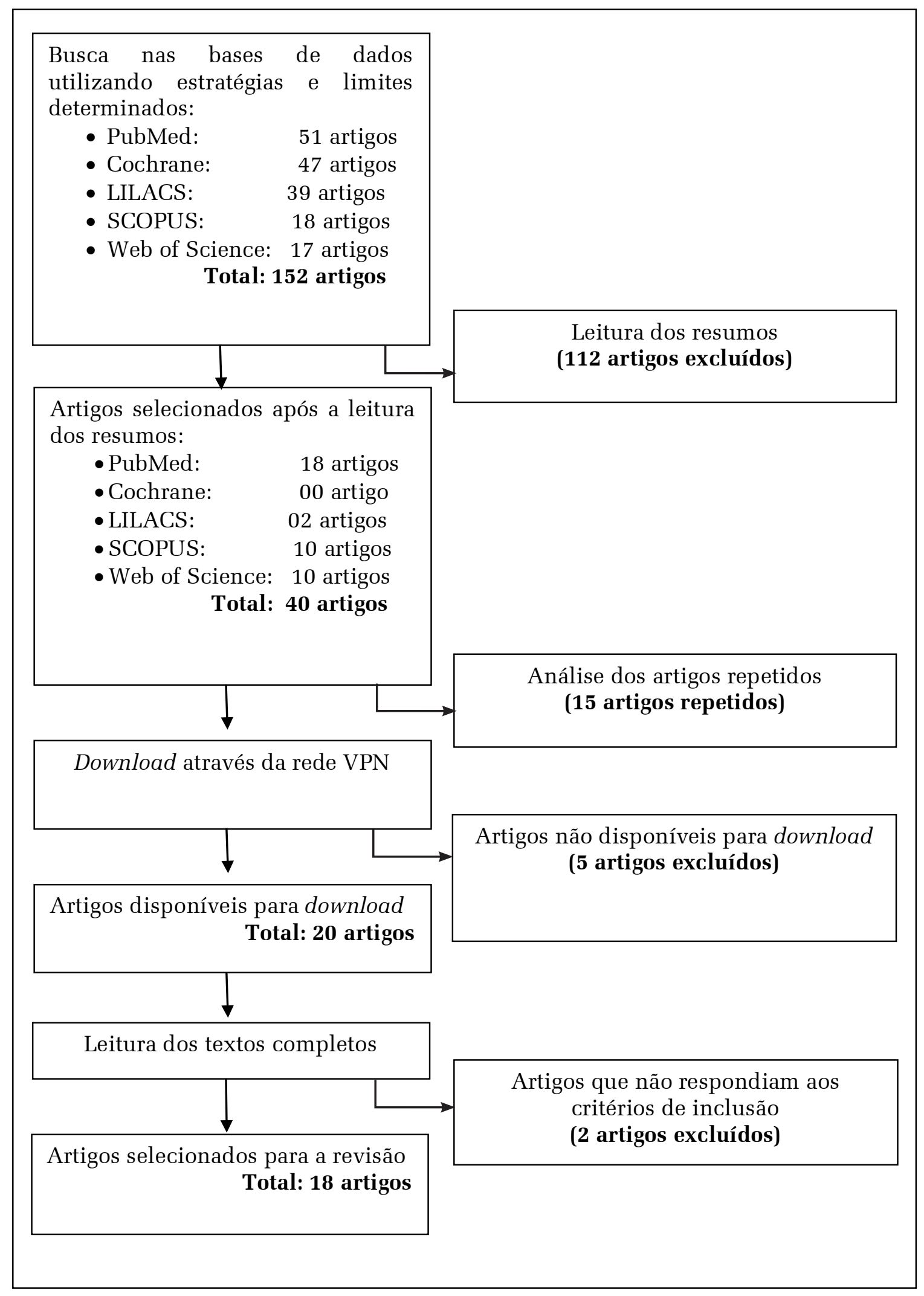

Figura 1 Esquema ilustrativo da estratégia utilizada para obtenção dos estudos incluídos na revisão sistemática 
Quadro 2 Características principais dos 20 artigos selecionados para leitura do texto completo e que se encontravam disponíveis para download

\begin{tabular}{|c|c|c|c|c|c|}
\hline Autoria & Ano & País & Tipo de estudo & População estudada & Objetivos \\
\hline $\begin{array}{l}\text { Christoffersen } \mathrm{T} \text {, } \\
\text { Olsen } \mathrm{EG}^{16}\end{array}$ & 1993 & Noruega & $\begin{array}{l}\text { Coorte } \\
\text { retrospectiva }\end{array}$ & $\begin{array}{l}29 \text { pacientes expostos a trauma } \\
\text { químico ocular por exposição à } \\
\text { bile de peixe ( } 28 \text { eram pescado- } \\
\text { res comerciais profissionais) }\end{array}$ & $\begin{array}{c}\text { Avaliar a severidade da lesão cau- } \\
\text { sada pela bile de peixe nos olhos } \\
\text { humanos e estimar a relevância } \\
\text { epidemiológica dessa lesão }\end{array}$ \\
\hline $\begin{array}{l}\text { Wong L, Ho SC, } \\
\text { Coggon D, Macdon- } \\
\text { ald DM, Cruddas } \\
\text { AM, Hwang CH, et } \\
\text { al. }^{17}\end{array}$ & 1993 & China & $\begin{array}{l}\text { Corte } \\
\text { transversal }\end{array}$ & $\begin{array}{c}\text { Comunidades pesqueiras de } \\
\text { Hong Kong }\end{array}$ & $\begin{array}{l}\text { Avaliar se a catarata está associa- } \\
\text { da a uma maior exposição à luz } \\
\text { solar e se os agentes antioxidan- } \\
\text { tes atuam protegendo contra ela }\end{array}$ \\
\hline $\begin{array}{l}\text { Schein OD, West S, } \\
\text { Muñoz B, Vitale S, } \\
\text { Maguire M, Taylor } \\
\text { HR, et al. }{ }^{18}\end{array}$ & 1994 & EUA & $\begin{array}{c}\text { Coorte } \\
\text { prospectiva }\end{array}$ & $\begin{array}{c}437 \text { pescadores comerciais da } \\
\text { Baía de Chesapeake }\end{array}$ & $\begin{array}{l}\text { Analisar a distribuição da opacifi- } \\
\text { cação cortical do cristalino e sua } \\
\text { relação com a exposição ocupa- } \\
\text { cional à radiação ultravioleta }\end{array}$ \\
\hline $\begin{array}{l}\text { Javitt JC, Taylor } \\
\text { HR }^{19}\end{array}$ & 1995 & EUA & $\begin{array}{l}\text { Revisão de } \\
\text { literatura }\end{array}$ & $\begin{array}{l}\text { Variável. Incluía a análise de dois } \\
\text { estudos que avaliaram popula- } \\
\text { ções de pescadores comerciais }\end{array}$ & $\begin{array}{l}\text { Avaliar: as variáveis físicas } \\
\text { e geográficas que afetam a } \\
\text { entrada dos raios UV no olho; as } \\
\text { evidências epidemiológicas que } \\
\text { associam a catarata à exposição } \\
\text { aos raios UV; e a efetividade das } \\
\text { barreiras de proteção na redução } \\
\text { da exposição ocular aos raios UV }\end{array}$ \\
\hline Taylor HR ${ }^{20}$ & $\begin{array}{l}1994- \\
1995\end{array}$ & Austrália & $\begin{array}{l}\text { Revisão de } \\
\text { literatura }\end{array}$ & $\begin{array}{l}818 \text { pescadores comerciais da } \\
\text { Baia de Chesapeake e } 336 \text { traba- } \\
\text { Ihadores rurais de Maryland }\end{array}$ & $\begin{array}{l}\text { Avaliação da associação entre } \\
\text { catarata e exposição aos raios } \\
\text { UVB, levando em consideração } \\
\text { os fatores individuais }\end{array}$ \\
\hline $\begin{array}{l}\text { Penland RL, Boniuk } \\
\text { M, Wilhelmus KR } 21\end{array}$ & 2000 & EUA & $\begin{array}{l}\text { Relato de caso } \\
\text { e revisão de } \\
\text { literatura }\end{array}$ & $\begin{array}{c}\text { Um pescador comercial/indiví- } \\
\text { duos com olhos infectados pela } \\
\text { Vibrio }\end{array}$ & $\begin{array}{c}\text { Descrever a epidemiologia da } \\
\text { infecção ocular pela Vibrio }\end{array}$ \\
\hline $\begin{array}{l}\text { Feretis E, Theodo- } \\
\text { rakopoulos P, Va- } \\
\text { rotsos } \mathrm{C} \text {, Efstathiou } \\
\mathrm{M} \text {, Tzanis C, Xirou } \\
\mathrm{T} \text {, et al. }{ }^{22}\end{array}$ & 2002 & Grécia & $\begin{array}{l}\text { Corte } \\
\text { transversal }\end{array}$ & $\begin{array}{c}\text { Moradores de } 3 \text { regiões subur- } \\
\text { banas de Atenas e } 2 \text { grupos de } \\
\text { monges/freiras e pescadores } \\
\text { comerciais }\end{array}$ & $\begin{array}{c}\text { Estimar a prevalência de lesões } \\
\text { oculares em função dos parâme- } \\
\text { tros de exposição à radiação UVB } \\
\text { e poluição do ar }\end{array}$ \\
\hline Vojnikovic B ${ }^{23}$ & 2007 & Croácia & $\begin{array}{l}\text { Estudo de corte } \\
\text { transversal }\end{array}$ & $\begin{array}{l}\text { Grupo: agricultores e pescadores } \\
\text { (1300 indivíduos)/Grupo 2: pes- } \\
\text { soas urbanas (71 indivíduos) }\end{array}$ & $\begin{array}{c}\text { Estimar em qual porcentagem a } \\
\text { retina periférica está afetada na } \\
\text { Degeneração Macular Relaciona- } \\
\text { da à Idade (DMRI) }\end{array}$ \\
\hline $\begin{array}{l}\text { Vojnikovic B, Njiric } \\
\text { S, Coklo M, Spanjol } \\
\mathrm{J}^{24}\end{array}$ & 2007 & Croácia & $\begin{array}{l}\text { Corte } \\
\text { transversal }\end{array}$ & $\begin{array}{c}\text { Grupo: agricultores e pescadores } \\
\text { (1300 indivíduos)/Grupo 2: pes- } \\
\text { soas urbanas (71 indivíduos) }\end{array}$ & $\begin{array}{c}\text { Estimar a correlação entre a } \\
\text { incidência de DMRI e exposição } \\
\text { solar na Ilha de Rab (Croácia) }\end{array}$ \\
\hline $\begin{array}{l}\text { Vojnikovic B, Njiric } \\
\text { S, Coklo M, Toth I, } \\
\text { Spanjol J, Marinovic } \\
\mathrm{M}^{25}\end{array}$ & 2007 & Croácia & $\begin{array}{l}\text { Corte } \\
\text { transversal }\end{array}$ & $\begin{array}{c}\text { Grupo: agricultores e pescadores } \\
\text { (480)/Grupo 2: pessoas urbanas } \\
\text { (61 indivíduos) }\end{array}$ & $\begin{array}{l}\text { Estimar a correlação entre } \\
\text { fatores climáticos, especialmen- } \\
\text { te exposição crônica à luz UV, e } \\
\text { o aparecimento de pterígio ou } \\
\text { síndrome de esfoliação }\end{array}$ \\
\hline
\end{tabular}

(Continua) 
Quadro 2 Continuação...

\begin{tabular}{|c|c|c|c|c|c|}
\hline Autoria & Ano & País & Tipo de estudo & População estudada & Objetivos \\
\hline $\begin{array}{l}\text { Novalbos J, Nogue- } \\
\text { roles P, Soriguer M, } \\
\text { Piniella F }{ }^{26}\end{array}$ & 2008 & Espanha & $\begin{array}{l}\text { Corte } \\
\text { transversal }\end{array}$ & 247 pescadores comerciais & $\begin{array}{c}\text { Avaliar as condições de saúde, } \\
\text { segurança e trabalho do setor } \\
\text { pesqueiro da Andaluzia (Espa- } \\
\text { nha) }\end{array}$ \\
\hline $\begin{array}{l}\text { Inchingolo F, Ta- } \\
\text { tullo M, Abenavoli } \\
\text { FM, Inchingolo AD, } \\
\text { Inchingolo AM, } \\
\text { Dipalma G }{ }^{27}\end{array}$ & 2010 & Itália & Relato de caso & Um pescador comercial & $\begin{array}{l}\text { Relatar um caso de acidente } \\
\text { ocular com gancho de pesca }\end{array}$ \\
\hline $\begin{array}{l}\text { Levy BS, Nassetta } \\
\mathrm{WJ}^{28}\end{array}$ & 2011 & EUA & $\begin{array}{l}\text { Revisão de } \\
\text { literatura }\end{array}$ & $\begin{array}{l}\text { Pessoas expostas a vazamen- } \\
\text { tos de óleo no mar, incluindo } \\
\text { pescadores comerciais e não } \\
\text { comerciais }\end{array}$ & $\begin{array}{l}\text { Revisar os efeitos adversos da ex- } \\
\text { posição aos vazamentos de óleo } \\
\text { e estabelecer um formulário- } \\
\text {-padrão para acompanhamento } \\
\text { médico dos indivíduos expostos }\end{array}$ \\
\hline $\begin{array}{l}\text { Rios AO, Rego RCF, } \\
\text { Pena P29 }\end{array}$ & 2011 & Brasil & $\begin{array}{l}\text { Revisão de } \\
\text { literatura }\end{array}$ & Pescadores comerciais & $\begin{array}{l}\text { Revisar os fatores de risco para } \\
\text { doenças e agravos relacionados à } \\
\text { pesca, excetuando-se acidentes }\end{array}$ \\
\hline $\begin{array}{l}\text { Tran KA, Green M, } \\
\text { Camuglia J, O’Ha- } \\
\text { gan } \mathrm{S}^{30}\end{array}$ & 2011 & Austrália & Relato de caso & $\begin{array}{l}\text { Um pescador comercial de } \\
\text { lagostas }\end{array}$ & $\begin{array}{c}\text { Relatar o caso de uma lesão } \\
\text { ocular causada pela antena de } \\
\text { lagosta }\end{array}$ \\
\hline $\begin{array}{l}\text { Agrawal R, Laude A, } \\
\text { Taneja } \mathrm{M}^{31}\end{array}$ & 2012 & Índia & $\begin{array}{c}\text { Relatos de } \\
\text { casos e revisão } \\
\text { de literatura }\end{array}$ & Dois pescadores comerciais & $\begin{array}{l}\text { Relatar dois casos de trauma } \\
\text { ocular por gancho de pesca e } \\
\text { revisar a literatura relacionada }\end{array}$ \\
\hline $\begin{array}{l}\text { Percin F, Akyol O, } \\
\text { Davas A, Saygi H }{ }^{7}\end{array}$ & 2012 & Turquia & $\begin{array}{l}\text { Corte } \\
\text { transversal }\end{array}$ & $\begin{array}{c}1166 \text { pescadores comerciais de } \\
\text { pequena escala do Mar Egeu }\end{array}$ & $\begin{array}{c}\text { Avaliar as condições de saúde, } \\
\text { segurança e trabalho dos pesca- } \\
\text { dores de pequena escala da costa } \\
\text { do Mar Egeu }\end{array}$ \\
\hline $\begin{array}{l}\text { Vivó JN, Hernández } \\
\mathrm{NH} \text {, Sanz AM }{ }^{32}\end{array}$ & 2012 & Cuba & Relato de caso & Um pescador comercial artesanal & $\begin{array}{c}\text { Relatar o caso de uma neoplasia } \\
\text { intraepitelial corneo-conjuntival } \\
\text { num pescador comercial ( } 20 \\
\text { anos de profissão e } 81 \text { anos de } \\
\text { idade) }\end{array}$ \\
\hline
\end{tabular}

\section{Discussão}

Encontrou-se ampla variedade de lesões oculares em trabalhadores da pesca, provindas da exposição a diversos riscos ocupacionais: físicos, químicos, biológicos e de acidentes. De modo a facilitar a análise, a discussão foi organizada de acordo com os resultados mais frequentes identificados nos artigos: lesões por exposição solar, traumas oculares, problemas oftalmológicos e/ou queixas oculares e fatores de proteção.

Lesões por exposição solar/raios ultravioleta do tipo B (UVB)

A catarata/opacificação do cristalino foi a principal lesão relacionada à exposição aos raios ultravioleta (UV) na atividade pesqueira. Realizados essencialmente na primeira metade da década de 1990, os estudos sobre essas patologias visavam principalmente a caracterizar o nexo causal entre exposição solar e catarata a partir dos comportamentos individuais e das histórias ocupacionais dos pescadores. Inicialmente, tais estudos encontraram dificuldades (e.g. viés de memória) para realização da aferição da exposição e estabelecimento de uma escala de severidade para a catarata, o que se refletiu nos primeiros resultados não apresentarem significância estatística para a relação entre maior exposição solar e níveis mais severos de catarata $^{33}$. Conhecendo os vieses supracitados, trabalhos subsequentes demonstraram que uma maior exposição ocupacional estava relacionada com uma maior incidência de opacificação $\operatorname{cortical}^{20}$ e 
que, anatomicamente, os quadrantes oculares mais acometidos eram os inferiores (i.e., menos protegidos pelas barreiras oculares), reforçando o papel da exposição direta à radiação solar como fator de risco para lesões desse tipo ${ }^{18}$. Tais estudos foram bastante úteis para as pesquisas seguintes, pois estabeleceram: a não existência de associação entre a exposição aos raios ultravioleta do tipo B (UVB) e a catarata nuclear, bem como entre a exposição aos raios ultravioleta do tipo A (UVA) e a catarata em geral; a importância da inclusão da aferição da exposição individual à radiação solar, extrapolando a análise para além dos estudos ecológicos; e, a necessidade de avaliação da relação entre a radiação ultravioleta e os danos oculares, especialmente a catarata e a degeneração macular senis ${ }^{20}$.

Estudos com pescadores e agricultores da Ilha de Rab, na Croácia, demonstraram que a ocorrência de degeneração macular relacionada à idade (DMRI) estava significativamente correlacionada à exposição crônica à luz solar e à síndrome de esfoliação ${ }^{25}$. Ainda, demonstraram que em uma significativa porcentagem da amostra, na presença de DMRI, não apenas a mácula se encontrava lesada como também a periferia da retina, sugerindo assim a "retinopatia relacionada à idade" como nomenclatura mais apropriada $^{23}$. Num outro desenho de estudo, o mesmo autor sugeriu uma relação causal entre a exposição solar crônica e a ocorrência de pterígio e/ou síndrome de esfoliação; no entanto, os vieses presentes comprometeram a confiabilidade dos resultados ${ }^{23}$.

A neoplasia intraepitelial córneo-conjuntival foi relacionada com a exposição prolongada à radiação UV em um relato de caso de um pescador artesanal ${ }^{32}$. Num estudo realizado na Grécia ${ }^{22}$, foram descritas as seguintes frequências relativas de lesões oculares nos pescadores estudados: 60\% possuíam lesões da conjuntiva; 50\% lesões da córnea; 10\% tinham lesões do cristalino; e menos de 5\% tinham lesões na pálpebra. Entretanto, as lesões em si não foram descritas, a correlação com exposição solar não foi conclusiva e o número absoluto de pescadores que compuseram a amostra não foi citado.

\section{Traumas oculares}

A Organização Internacional do Trabalho reconhece os acidentes como o principal problema de saúde relacionado à atividade da pesca ${ }^{34}$. Em concordância com essa afirmação, o trauma ocular decorrente de acidentes foi a segunda injúria ocular mais relatada, tendo sido abordada em seis artigos (33\%), embora esses trabalhos se tratavam essencialmente de relatos de casos. Os traumas oculares mais relatados foram as lacerações da pálpebra superior por perfuração pelo gancho de pesca ${ }^{28}$, sendo que em um dos casos a córnea também foi lacerada. O contato dos olhos com fragmentos ou secreções de animais marinhos, como peixes, ostras e lagostas, também foi descrito como uma importante causa de traumatismo ocular, levando ao blefaroespasmo, abscesso conjuntival ou até mesmo úlcera corneana com evolução para opacidade corneana e limitação da visão ${ }^{16,21,30}$.

Essa importante representatividade dos acidentes entre as causas de morbidades oftalmológicas consubstancia os achados em estudo que afirmou que os pescadores trabalham em ambientes hostis e instáveis, se encontrando expostos a fatores de riscos, como o manuseio de peixes e frutos do mar e o consumo exacerbado de álcool, que os tornam mais vulneráveis à ocorrência de acidentes ${ }^{11}$. De modo complementar, outro trabalho afirmou que os pescadores trabalham em período integral, incluindo fins de semana e turnos noturnos, e, assim, incrementam o tempo de exposição aos acidentes ${ }^{12}$. A Organização Internacional do Trabalho também relaciona tais ocorrências com outras características dessa ocupação, como: a necessidade de alto grau de coordenação entre a equipe que realiza o lançamento/retirada do equipamento de pesca do mar/rio; o manuseio de instrumental pesado; ou a pressão de tempo imposta pelas leis de proteção aos recursos naturais, que limitam o período do ano permitido à pesca, fazendo que os pescadores sejam impelidos a fazer uso máximo desse tempo, independentemente das condições climáticas. Essa realidade foi numericamente demonstrada por um estudo que encontrou, dentre os motivos de busca por atendimento médico para pescadores, uma taxa de $35 \%$ correspondente a acidentes ${ }^{9}$.

Ainda no grupo dos acidentes, uma revisão de literatura $^{28}$ elencou as manifestações clínicas em pessoas expostas a vazamentos de óleo no mar, incluindo pescadores comerciais e não comerciais. Os olhos são principalmente atingidos pelos vapores das substâncias voláteis ou pelo contato direto com a água. O espectro de manifestações oculares foi variado, constando de sinais e sintomas inespecíficos, como prurido, hiperemia e irritação, e a conjuntivite. Os agentes causadores dessas manifestações fazem parte de uma mistura química composta por benzeno, tolueno, etilbenzeno e xilenos, bem como mercúrio e vanádio, em alguns casos.

\section{Problemas oftalmológicos e/ou queixas oculares}

Nos últimos cinco anos, alguns estudos que avaliavam de modo holístico a saúde do pescador foram realizados. Em 2008, um trabalho ${ }^{26}$ relatou que os "problemas oculares e oftalmológicos" foram a segunda condição médica mais relatada (38\%) pelos 247 pescadores no inquérito epidemiológico 
realizado, atrás apenas dos "problemas de pele", com 54\%. No entanto, em ambos os casos, não foram especificados os "problemas" referidos pelos entrevistados. De modo similar, uma revisão de literatura ${ }^{29}$ relata outros estudos que encontraram "queixas oculares" e até mesmo quantificam essas queixas, referindo que $41,8 \%$ dos pescadores possuíam problemas oftalmológicos; porém, as queixas e problemas não são especificados.

Em 2012, um estudo ${ }^{7}$ aplicou randomicamente um questionário que avaliava o estado de saúde (no último ano) dos 1.166 pescadores da amostra. Todas as queixas e doenças reportadas eram confirmadas e classificadas por meio da Classificação Estatística Internacional de Doenças e Problemas Relacionados com a Saúde (CID), por dois médicos. Dessa forma, obteve-se a ocorrência de patologias oculares em $41 \%$ dos entrevistados. Em termos absolutos, foram 471 casos confirmados, sendo 385 transtornos de refração e acomodação (CID H52), 55 casos de conjuntivite (CID H10.1) e 31 casos de inflamações da pálpebra (CID H01). Os dados estatísticos produzidos por essas pesquisas evidenciam quais queixas e morbidades afligem os trabalhadores da pesca de acordo, basicamente, com a localização anatômica e, com isso, permitem a visualização de quais grupos de patologias devem ser priorizados nos estudos voltados para essas populações. Nesse sentido, os achados supracitados clarificam a relevância das doenças oftalmológicas enquanto causa de morbidade nos trabalhadores da pesca, justificando a realização de estudos mais apurados e específicos para tais acometimentos nesses indivíduos.

\section{Fatores de proteção}

A existência e eficácia dos mecanismos de proteção para os fatores de riscos mais comumente relacionados aos danos oftalmológicos associados à profissão da pesca - como a exposição aos raios UVB, os acidentes e o contato com animais marinhos - demonstra que a ocorrência dessas lesões continua a ser quantitativamente relevante, pois as medidas cabíveis para a reversão desse quadro não estão sendo tomadas em diferentes níveis - desde o individual, com o não uso dos equipamentos de proteção individual, até o nível coletivo, exemplificado pela inexistência/escassez de programas de educação em saúde e políticas públicas direcionados para esse público. Essa carência de medidas sanitaristas já havia sido identificada por Grainger desde 1993, quando afirmou que eram necessários programas de treinamento para os pescadores que pudessem reduzir as exposições aos riscos preveníveis da profissão, ressaltando que muitas comunidades pesqueiras pertencem às minorias étnicas e são economicamente desfavorecidas - consequentemente necessitam de maior suporte por parte dos órgãos públicos ${ }^{11}$; similarmente, outro trabalho ${ }^{12}$ ressaltou a importância da realização de medidas para a prevenção de quedas e acidentes relacionados com máquinas, por meio do encapamento destas, da instalação de pisos antiderrapantes e da fixação das escadas. Para além das morbidades, o não uso de EPIs pode afetar de modo importante a mortalidade dos pescadores, como demonstrado num estudo estadunidense ${ }^{35}$.

No que concerne à prevenção primária, a maioria dos estudos indicou o uso de chapéus com aba, óculos, óculos escuros, bem como a redução da exposição solar, se possível, como medidas preventivas eficazes $^{20,25,27-31}$; por outro lado, não houve redução do risco de catarata nos indivíduos com índices séricos mais altos de antioxidantes, ou seja, em uso de vitaminas e afins ${ }^{17}$. Entretanto, uma pesquisa ${ }^{7}$ encontrou que de 1.166 pescadores entrevistados, 85\% relataram incômodo ocular em razão do reflexo da luz solar na água do mar. Não obstante, apenas $34 \%$ desses utilizavam óculos escuros como proteção. Em termos de prevenção secundária, a lavagem imediata dos olhos ${ }^{16}$, a não mobilização do agente perfurante e o encaminhamento imediato para um atendimento emergencial em ambiente hospitalar foram indicados como importantes variáveis para um desfecho mais favorável ${ }^{31}$. Nos níveis da promoção e da gestão em saúde, as medidas educativas sobre modos de vida saudáveis e prevenção de acidentes também foram memoradas, bem como o imperativo para a implementação de políticas de saúde específicas e assistência especializada para os trabalhadores da pesca ${ }^{7,26,28,30}$. Seis artigos não citaram qualquer informação sobre fatores de proteção ${ }^{18,21-24,32}$.

Ademais, vale destacar que, devido à escassez de estudos sobre o tema, foram incluídos nesta revisão artigos com variados desenhos de estudo, o que limitou a análise comparativa dos trabalhos encontrados. A maioria dos artigos obtidos foi do tipo corte transversal ou relato de caso (67\%) - desenhos de estudo que não são os mais indicados para o estabelecimento de associação causal entre a pesca comercial e as lesões oculares.

Há uma significativa incidência e variedade de lesões oculares relacionadas ao exercício da pesca comercial, compreendida em sua ampla diversidade. Paradoxalmente, há pouco reconhecimento desse fato por parte dos órgãos públicos na maioria dos países. Dessa maneira, essas patologias continuam a ter um importante impacto sobre a morbidade dessas populações. Por conseguinte, essa morbidade se reflete em dias de trabalhos perdidos ou até mesmo incapacidade permanente para o exercício profissional da pesca, o que tem importantes implicações sociais, uma vez que o perfil socioeconômico dessas populações é reconhecidamente de extrema 
fragilidade. Por essa razão, estudos epidemiológicos bem conduzidos, que possam caracterizar o estado de saúde dos pescadores, incluindo sua saúde ocular - a exemplo do realizado na Turquia ${ }^{7}$ - devem ser incentivados nos demais países. Além disso, faz-se necessária a investigação dos motivos da não adesão desses profissionais ao uso dos equipamentos de proteção individual, que pode ser em grande parte decorrente do processo de trabalho imposto a esses trabalhadores, tais como grandes jornadas de trabalho, sem pausas e com baixa remuneração, o que leva ao adoecimento. A realização de mais estudos nesse grupo ocupacional pode gerar informações importantes, servindo de base para a elaboração de políticas de saúde que reconheçam as especificidades do trabalhador da pesca.

\section{Conclusões}

Os estudos encontrados sugerem que o exercício da pesca comercial se relaciona com uma maior ocorrência de determinadas lesões oculares. Dentre estas, a catarata e a degeneração macular relacionada à idade - ambas diretamente ligadas a uma maior exposição solar - foram as mais associadas à profissão. Os traumas oculares por acidentes de mecanismos variados (penetrante, químico etc.) representaram a segunda categoria mais frequentemente citada.
Esses acidentes foram relacionados com fatores de risco, como o manuseio de peixes e frutos do mar, o etilismo e as jornadas prolongadas de trabalho.

As medidas de proteção mais recomendadas como prevenção primária foram o uso de equipamentos de proteção individual, como óculos (escuros ou "de proteção") e chapéus com abas, e a redução do tempo de exposição à luz solar. Além disso, faz-se necessária a investigação dos motivos da não adesão dos pescadores profissionais ao uso dos EPIs. Ademais, é de fundamental importância que os órgãos públicos sejam implicados na garantia dos ajustes dos processos de trabalho dos pescadores, em termos de propiciar-lhes melhores condições de trabalho, como aplicação das medidas de segurança no ambiente de trabalho, ajuste de cargas horárias etc.

De acordo com os achados aqui descritos, a maioria das doenças oftalmológicas relacionadas ao trabalho da pesca são preveníveis, o que indica a necessidade de implementação de programas de educação e políticas de saúde, ambos especificamente direcionados para a população pesqueira. Nesse ínterim, apesar dos variados tipos de estudo encontrados, notabilizou-se uma escassez de estudos adequados à análise de causalidade (corte transversal, coortes etc). O desenvolvimento desse tipo de estudo se faz fundamental para que os prováveis nexos causais possam ser consolidados, dando maior suporte técnico às políticas de proteção ao pescador comercial.

\section{Contribuições de autoria}

Freitas da Silva D: contribuiu substancialmente no projeto e em seu delineamento, levantamento de dados, análise, interpretação e elaboração da versão final do manuscrito, além da importante colaboração na revisão crítica. Tavares-Neto J: participou do projeto e em seu delineamento, bem como na sua revisão crítica. Rêgo RF: participou do projeto e em seu delineamento, com contribuição importante na sua revisão crítica e na aprovação final da versão publicada.

\section{Referências}

1. Food and Agriculture Organization of the United States. Glossary. 2016 [Citado em 2016 jan 10]. Disponível em: http:/www.fao.org/faoterm/ en/?defaultCollId=21www.fao.org/fi/glossary/

2. Brasil. Lei $n^{0}$ 9.605, de 12 de fevereiro de 1998. Dispõe sobre as sanções penais e administrativas derivadas de condutas e atividades lesivas ao meio ambiente, e dá outras providências. Diário Oficial da União, Brasília, DF, 12 jan. 1998, Seção I, p. 1 [Citado em 2013 jan 10]. Disponível em: http:// www.planalto.gov.br/ccivil_03/Leis/L9605.htm

3. Organização Internacional do Trabalho. [Dispõe sobre informações gerais sobre a pesca]. 2011 [Citado em 2016 abr. 22]. Disponível em: http:// www.iloencyclopaedia.org/part-x-96841/fishing
4. Brasil. Ministério da Agricultura, Pecuária e Abastecimento. Aquicultura e pesca. 2016 [Citado em 2016 nov 17]. Disponível em: http://www. agricultura.gov.br/aquicultura-pesca

5. Brasil. Ministério da Pesca e Aquicultura. Boletim Estatístico da Pesca e Aquicultura. 2011 [Citado em 2016 nov 18]. Disponível em: http://www.icmbio. gov.br/cepsul/images/stories/biblioteca/download/ estatistica/est_2011_bol_bra.pdf

6. Brasil. Lei $\mathrm{n}^{\mathrm{O}} 11.959$, de 29 de junho de 2009. Dispõe sobre a Política Nacional de Desenvolvimento Sustentável da Aquicultura e da Pesca, regula as atividades pesqueiras, revoga a Lei $\mathrm{n}^{\circ}$ 7.679, de 23 de novembro de 1988. Brasília, DF, 29 jun. 2009 [Citado em 2013 jan 10]. Disponível 
em: http://www.planalto.gov.br/ccivil_03/_ato20072010/2009/Lei/L11959.htm

7. Percin F, Akyol O, Davas A, Saygi H. Occupational health of Turkish Aegean small-scale fishermen. Occup Med (Lond). 2012 Mar [Citado em 2013 jan 12];62(2):148-51. Disponível em: http://www.ncbi. nlm.nih.gov/pubmed/22113895

8. Lawrie T, Matheson C, Ritchie L, Murphy E, Bond C. The health and lifestyle of Scottish fishermen: a need for health promotion. Health Educ Res. 2004 Ago [Citado em 2013 jan 12];19(4):373-9. Disponível em: http://www.ncbi.nlm.nih.gov/ pubmed/15199010

9. Matheson C, Morrison S, Murphy E, Lawrie T, Ritchie L, Bond C. The health of fishermen in the catching sector of the fishing industry: a gap analysis. Occupational medicine Oxford England. 2001 Ago [Citado em 2013 fev 13];51(5):305-11. Disponível em: http://occmed.oxfordjournals.org/ content/51/5/305.full.pdf

10. Reilly MS. Mortality from occupational accidents to United Kingdom fishermen 1961-80. Br J Ind Med. 1985;42(12):806-14.

11. Grainger CR. Hazards of commercial fishing. Word Health Forum. 1993;14(3):313-5.

12. Bull N, Riise T, Moen BE. Occupational injuries to fisheries workers in Norway reported to insurance companies from 1991 to 1996. Occup Med (Lond). 2001;51(5):299-304.

13. Grimsmo-Powney H, Harris EC, Reading I, Coggon D. Occupational health needs of commercial fishermen in South-West England. Occup Med (Lond). 2010 Jan [Citado em 2013 fev 20];60(1):4953. Disponível em: http://eprints.soton. ac.uk/150203

14. Kreis AJ. Fishing Down Under: case report and review of management of a fishhook injury of the eyelid. Clin Exp Optom. 2008 Set [Citado em 2013 jan 20]; 91(5):473-5. Disponível em: http://www. ncbi.nlm.nih.gov/pubmed/18430035

15. Alfaro III DV, Jablon EP, Rodriguez Fontal M, Villalba SJ, Morris RE, Grossman M, et al. Fishing-related ocular trauma. Am J Ophthalmol. 2005 Mar [Citado em 2012 dez 20];139(3):48892. Disponível em: http://www.sciencedirect. com/science/article/B6VK5-4FBW62Y-3/2/ ff277dbc822483d4ecd807cee9041dad

16. Christoffersen T, Olsen EG. Injury to the cornea due to fish bile. Scand J Work Environ Health. 1993 Oct [Citado em 2013 jan 15];19(5):358-9. Disponível em: http://www.sjweh.fi/show_abstract. php?abstract_id=1462

17. Wong L, Ho SC, Coggon D, Macdonald DM, Cruddas AM, Hwang CH, et al. Sunlight exposure, antioxidant status, and cataract in Hong Kong fishermen. J Epidemiol Community Health. 1993;47:46-9.

18. Schein OD, West S, Muñoz B, Vitale S, Maguire M, Taylor HR, et al. Cortical lenticular opacification: distribution and location in a longitudinal study. Invest Ophthalmol Vis Sci. 1994;35(2):363-6.

19. Javitt JC, Taylor HR. Cataract and latitude. Doc Ophthalmol. 1995;88:307-25.

20. Taylor HR. Ocular effects of UV-B exposure. Doc Ophthalmol. 1994-1995 [Citado em 2013 jan 19];88(3-4):285-93. Disponível em: http://www. ncbi.nlm.nih.gov/pubmed/7634997

21. Penland RL, Boniuk M, Wilhelmus KR. Vibrio ocular infections on the U.S. Gulf Coast. Cornea. 2000 Jan [Citado em 2013 jan 19];19(1):26-9. Disponível em: http://www.ncbi.nlm.nih.gov/ pubmed/10632004

22. Feretis E, Theodorakopoulos P, Varotsos C, Efstathiou M, Tzanis C, Xirou T, et al. On the plausible association between environmental conditions and human eye damage. Environ Sci Pollut R. 2002;9(3):163-5.

23. Vojniković B. Age-related macular degeneration is not macular process only: peripheral retina is attacked too. Coll Antropol. 2007 Jan [Citado em 2013 jan 17];31 Suppl 1:3-5. Disponível em: <http://www.ncbi.nlm.nih.gov/ pubmed/17469740>. Acesso em 17 de Janeiro de 2013.

24. Vojniković B, Njirić S, Coklo M, Spanjol J. Ultraviolet sun radiation and incidence of agerelated macular degeneration on Croatian Island Rab. Coll Antropol. 2007 Jan [Citado em 2013 jan 17];31 Suppl 1:43-4. Disponível em: http://www. ncbi.nlm.nih.gov/pubmed/17469748

25. Vojniković B, Njirić S, Coklo M, Toth I, Spanjol J, Marinović M. Sunlight and incidence of pterygium on Croatian Island Rab: epidemiological study. Coll Antropol. 2007 Jan [Citado em 2013 jan 17];31 Suppl 1:61-2. Disponível em: http://www.ncbi.nlm. nih.gov/pubmed/17469753

26. Novalbos J, Nogueroles P, Soriguer M, Piniella F. Occupational health in the Andalusian Fisheries Sector. Occup Med (Lond). 2008 Mar [Citado em 2013 jan 20];58(2):141-3. Disponível em: http:// www.ncbi.nlm.nih.gov/pubmed/18245787

27. Inchingolo F, Tatullo M, Abenavoli FM, Inchingolo AD, Inchingolo AM, Dipalma G. Fish-hook injuries: a risk for fishermen. Head Face Med. 2010 Jan [Citado em 2013 jan 26];6(1):28. Disponível em: http://www.pubmedcentral.nih.gov/articlerender.fc gi? artid $=3012659 \&$ tool $=$ pmcentrez\&rendertype $=$ abstract

28. Levy BS, Nassetta WJ. The adverse health effects of oil spills: a review of the literature and a framework for medically evaluating exposed individuals. Int J Occup Environ Health. 2011;17(2):161-7.

29. Rios AO, Rego RCF, Pena P. Doenças em trabalhadores da pesca. Rev Baiana Saude Publica. 2011;35(1):175-88.

30. Tran KA, Green M, Camuglia J, O’Hagan S. Penetrating eye injury from a crayfish antenna. Med J Aust. 2011 Dez [Citado em 2013 jan 
30];195(11/12):706-7. Disponível em: http:// www.mja.com.au/public/issues/195_11_121211/ tra10794_fm.html

31. Agrawal R, Laude A, Taneja M. Fish-hook injury of the eye. Int Ophthalmol. 2012 Jun [Citado em 2013 jan 29];32(3):269-71. Disponível em: http://www. ncbi.nlm.nih.gov/pubmed/22456795

32. Vivó JN, Hernández NH, Sanz AM. Neoplasia intraepitelial córneo-conjuntival: a propósito de un caso. Rev Méd Electrón. 2012 [Citado em 2013 jan 29]; Disponível em: http://www.revmatanzas.sld. cu/revista\%20medica/ano\%202012/vol4\%202012/ tema10.htm
33. Taylor HR, West SK, Rosenthal FS, Muñoz B, Newland HS, Abbey H, et al. Effect of ultraviolet radiation on cataract formation. $\mathrm{N}$ Engl J Med. 1988;319(22):1429-33.

34. Organização Internacional do Trabalho. [Dispõe sobre Problemas de Saúde e Padrões de Doença (em pescadores)]. 2011 [Citado em 2016 abr 29]. Disponível em: http://www.iloencyclopaedia.org/ part-x-96841/fishing/110-66-fishing/commercialfisheries-environmental-and-public-health-issues

35. Schnitzer PG, Landen DD, Russell JC. Occupational injury deaths in Alaska's fishing industry, 1980 through 1988. Am J Public Health. 1993;83(5):685-8. 